\title{
Design of high-performance energy integrator detector for wideband radar
}

\author{
CHANG Jiayun, FU Xiongjun*, JIANG Wen, and XIE Min
}

School of Information and Electronics, Beijing Institute of Technology, Beijing 100081, China

\begin{abstract}
Target detection for wideband radar has recently received extensive attention. The classical energy integrating (EI) detector will always accumulate excess clutter or noise energy, which leads to unacceptable performance deterioration if the detection window is not selected properly. In this paper, an El detector for the distributed targets in the Gaussian environment is proposed. First, at the stage of preparatory work, the target models are proposed, then, the problem formulation is introduced. Subsequently, in the aspect of optimizing the method of detection window search and the method of threshold setting, the detailed design stages of the proposed detector are provided. Furthermore, theoretical analyses show that the proposed detector is easy to hardware implementation, and it does not need the prior knowledge about the spatial distribution of the target scattering centers in practical radar detection application. Finally, the performance assessment conducted by Monte Carlo simulations verifies that the proposed detector outperforms the conventional detectors.
\end{abstract}

Keywords: radar, detection, distributed target, integration detector.

DOI: $10.21629 /$ JSEE.2019.06.07

\section{Introduction}

With the development and advancement of radar technology, apart from the detection and tracking of targets, the modern radar needs to class and recognize interested targets. The range resolution for low resolution radars (LRRs) is very low, and the energy of the echo signal is mostly concentrated in one range resolution cell, in this case, the target is regarded as a point-like target $[1,2]$. Therefore, LRRs cannot capture enough detail property of the target, and mainly serve for target detection and tracking. However, wideband high-resolution radars (HRRs) $[3,4]$ can spatially resolve a target into some scattering centers $[5,6]$. Hence, wideband HRRs have the capability to obtain more abundant and precise information of targets and

\footnotetext{
Manuscript received January 24, 2018.

*Corresponding author.

This work was supported by the National Natural Science Foundation of China (61571043) and the 111 Project of China (B14010).
}

environment, and widely applied in target classification, recognition, and imaging [7-13].

Due to the increasing of the range resolution for HRRs, energy in each range cell backscattered by distributed clutter is decreased, which can enhance the radar detection performance largely by appropriate detection strategies $[14,15]$. However, due to the leakage of the target energy to the reference cells, the conventional target detection algorithms of the narrowband radar, such as the constant false alarm ratio (CFAR) [16], fail for distributed targets [1720].

Therefore, it is very urgent to study the target detection algorithms for the wideband radar. At present, the main solutions of distributed target detection include the following: firstly, the mode of the narrowband detection and broadband tracking is utilized for detection of the distributed targets. However, the complexity of the system is remarkably increased. Thus, resorting to directly carrying out distributed target detection can be considered, and related studies have been extensively addressed. In [21], the statistical distribution of the maximal invariant was provided. In [22], the problem of adaptive detection in homogeneous Gaussian interference $[23,24]$ with the unknown covariance matrix was analyzed. However, under the situations of HRRs, the practical background clutter may no longer be modeled precisely as a Gaussian random variable (RV) [25]. In [26], two sets of training samples were exploited to resolve the radar detection problem in a homogeneous environment. In [27], the constrained maximum likelihood (ML) estimates were derived and the generalized likelihood ratio test (GLRT) [28,29] was designed to deal with the problem of detecting a distributed target embedded in homogeneous Gaussian. However, those detectors need some priori knowledge about the disturbance covariance matrix. In [30], an adaptive CFAR detector was designed to detect distributed targets in the clutterdominated heterogeneous environment. In [31 - 33], adaptive target detection approaches in a partially homogeneous 
Gaussian disturbance were proposed. However, those detectors are limited by the prior assumption that return signals are matched. In [34], it focused on the problem of fluctuating target detection in heavy-tailed K-distributed clutter. However, probability density function (pdf) of total noise is rather tedious owing to its complexity.

In the view of the fact that the energy integrating (EI) detector can deal with the detection of range-distributed targets, such as the target detection of Gaussian/non-Gaussian clutter pdf, and the uniform/non-uniform clutter power of each detection cell. However, the classical EI detector will always accumulate excess noise or clutter energy, which leads to unacceptable performance deterioration if the detection window is not selected properly. In this paper, a high performance EI detector by optimizing the detection threshold setting and search mode of the detection window is presented. Theory analysis indicates the proposed detector is non-dependent on the prior distribution information of target scattering centers, and simulation results show that the proposed detector outperforms the conventional detectors.

\section{Detection problem}

With the increase of range resolution for wideband radar, scattering centers of targets are distributed into a number of radar range cells. However, due to the change of movement attitude or occlusion, the strong scattering centers of the target may occupy only a fraction of resolution cells in the range profile. Assume that $K(1 \leqslant K \leqslant N)$ scattering centers of the distributed target, which is called the range extent of the target, occupy $N$ range resolution cells in the range profile, and different scattering centers are distributed on different range resolution cells. The detection problem of targets can be formulated in terms of the following binary hypotheses test $[35,36]$, that is,

$$
\left\{\begin{array}{l}
H_{0}: z=w \\
H_{1}: z=s+w
\end{array}\right.
$$

where $\boldsymbol{z}$ is the radar return signal, $s=\left\{s_{1}, s_{2}, \ldots, s_{N}\right\}$ is the target signal, and $\boldsymbol{w}=\left\{w_{1}, w_{2}, \ldots, w_{N}\right\}$ denotes the compound-Gaussian signals shown as

$$
\boldsymbol{c}=\sqrt{\tau} \boldsymbol{x}
$$

where $\boldsymbol{c}$ is an $N$-dimensional complex vector, $\boldsymbol{x} \sim$ $C N\left(0,2 M_{X}\right), M_{X}$ is the covariance matrix, named speckle, $\tau$ is the variance of the Gaussian vector, and represents the local power of the Gaussian signals. Under the $H_{0}$ hypothesis, the $\mathrm{N}$ th-order pdf can be given by

$$
p_{\boldsymbol{z} \mid H_{0}}\left(\boldsymbol{z} \mid H_{0}\right)=\frac{1}{(2 \pi)^{N}\left|\boldsymbol{M}_{X}\right|} h_{N}\left(\boldsymbol{z}^{\mathrm{H}} \boldsymbol{M}_{X}^{-1} \boldsymbol{z}\right)
$$

where $h_{N}(\cdot)$ indicates the conjugate transpose operator, and || indicates matrix determinant. $h_{N}$ is defined as

$$
h_{N} \triangleq \int_{0}^{\infty} \frac{1}{\tau^{m}} \exp \left(-\frac{\boldsymbol{x}}{2 \tau}\right) p_{\tau}(\tau) \mathrm{d} \tau
$$

where $p_{\tau}(\tau)$ is the pdf of $\tau$.

For a detection window with length $N$, the test statistics can be expressed by

$$
T(\boldsymbol{x})_{\text {INTEGRATOR }}=\frac{2}{\sigma^{2}} \sum_{n=1}^{N}\left|x_{n}\right|^{2} .
$$

Under the hypothesis of $H_{0}$, the test statistic obeys the centralized chi-squared distribution with $2 N$ degrees of freedom shown as follows:

$$
T(\boldsymbol{x})_{\text {INTEGRATOR }} \sim \chi_{2 N}^{2} .
$$

The relation between threshold $\lambda$ and false alarm probability $P_{f a}$ is

$$
P_{f a}=1-X_{0}(\lambda, 2 N)
$$

where $X_{0}(u, v)$ is the cumulative distribution function (CDF) for the central chi osquared distribution with $v$ degrees of freedom shown as

$$
X_{0}(u, v)=\int_{-\infty}^{u} \frac{1}{2^{\frac{v}{2}} \Gamma\left(\frac{v}{2}\right)} x^{\frac{v}{2}-1} \mathrm{e}^{-\frac{x}{2}} \mathrm{~d} x
$$

where for integer $J, \Gamma(N)=(N-1)$ !.

Under the hypothesis of $H_{1}$, the test statistic obeys the decentralized chi-squared distribution with $2 N$ degrees of freedom, and the non-central parameter is defined as follows:

$$
\eta=\frac{2 \sum_{n=1}^{N}\left|s_{n}\right|^{2}}{\sigma^{2}}
$$

Then, the detection probability is

$$
P_{d}=1-X_{1}(u ; \eta, 2 N)
$$

where $X_{1}(u ; \eta, 2 N)$ is a non-central chi-squared cumulative distribution function with a degree of freedom of $2 N$ and a non-central parameter is $\eta, X_{1}(u ; \eta, 2 N)$ can be written as

$$
X_{1}(u ; \eta, 2 N)=\int_{0}^{u} \frac{1}{2}\left(\frac{x}{\eta}\right)^{\frac{N-1}{2}} \mathrm{e}^{-\frac{1}{2}(x+\eta)} I_{\frac{N}{2}-1}(\sqrt{\eta x}) \mathrm{d} x
$$

where $I_{r}(u)$ is the Bessel function of the first kind with order $r$, and it is can be expressed as

$$
I_{r}(u)=\frac{\left(\frac{1}{2} u\right)^{2}}{\sqrt{\pi} \Gamma\left(r+\frac{1}{2}\right)} \int_{0}^{\pi} \mathrm{e}^{u \cos \theta} \sin ^{2 r} \theta \mathrm{d} \theta .
$$


According to the above analysis, we can derive the detection threshold

$$
\lambda=X_{0}^{-1}\left(1-P_{f a}, 2 N\right) .
$$

In many practical scenarios, the traditional EI detector pre-estimates the number $K$ of range-resolution cells occupied by distributed targets, and then selects the appropriate window length $N$ to accumulate the signal energy of the detected window. The window length $N$ should be approximately equal to the estimated value $K$, and the window sliding step can be as large as possible within the allowable range. The window corresponding to the maximum of test statistics is the detection window where the range distributed target is located. Assume that the central position of the detection window is called $P_{\text {mid }}$. Hence, the position of the target is given below:

$$
P_{\text {Tar }}=P_{\text {mid }}+\frac{N}{2} \Delta R
$$

where $\Delta R$ is the range resolution cell. In practice, however, the position or size of the range window is not always estimated accurately for the traditional EI detector. Therefore, the detector will accumulate the excess noise energy when it accumulates the energy of the scattering centers in the detection window, which results in the deterioration of the detection performance.

\section{Proposed detector}

When the wideband radar needs to detect the target in the whole range cells, it can utilize the sliding window and judge whether each window has the target. The window length should be approximately equal to the possible target length. However, if the range window for the EI detector is not properly selected, the traditional EI detector will accumulate excess noise energy and result in accumulation loss. Thus, the traditional EI detector is improved from two aspects in this paper: the search method of the detection window and the setting of the detection threshold. The novel search method of the detection window will investigate the radar's echoes in all the sliding windows with any possible length at any position of a given range gate. Thus, the position of the detection window is more precise, which is conducive to obtaining higher detection gain. In addition, during the actual detection application, the distribution of scattering points in different echoes will change with the transforms of target motion and radar angles of view, the test statistics will be not stable, thus the threshold will be varied. However, the traditional EI detector utilizes the fixed threshold to compare with the unstable test statistics of all the echoes, so its credibility and correctness of detection results will be poor. Through the optimized method of threshold setting, we will provide sets of adaptive thresholds changed with detection windows occupying different numbers of range cells. In this case, the problem that the actual length of the detection window, which is used to acquire the detection threshold, is not consistent with the window length occupied by test statistics of different echoes will be solved, and the detection threshold will be more appropriate. Hence, detection performance will be significantly improved.

\subsection{New search method of detection window}

The radar returns of all the sliding windows with any possible length at any position of a given range gate is investigated, and the sliding window with the largest average energy is regarded as the final detection window of the target for wideband radar, and here the search method is called the novel search method of the detection window for the proposed detector to distinguish that of the traditional EI detector. The design process of the EI detector using the novel window search method is composed of six parts and can be described as follows:

Step 1 The range of the sliding window length is set in advance. The minimum and maximum radial sizes of the target are assumed, and then the corresponding sampling points can be calculated respectively, which is the range of the sliding window length. Note that, the sliding window corresponding to the minimum number of points is called the minimum sliding window, which may not contain all scattering points of the range-distributed target.

Step 2 The current pulse data is simulated according to the observed signal model, and then pulse compression is performed (the noise-only data is included in the observed signal model to obtain the detection threshold).

Step 3 According to the step of position and sizes of the sliding window, the detection data of corresponding sliding windows (any possible position and the possible length of the sliding window in the range gate) are obtained, the average power of each sliding window is calculated. Take the sliding window corresponding to the maximum value as the detection window, and record its central position $P_{\text {mid }}$ and length $N$.

Step 4 The test statistics in the detection window are obtained by

$$
\Lambda=\frac{2}{\sigma^{2}} \sum_{n=1}^{N}\left|x_{n}\right|^{2} .
$$

Step 5 According to the Monte Carlo simulation times $T$, the Steps 2 to 4 are repeated, and then the test statistics of all sliding windows are obtained. Then, the detection threshold based on required $P_{f a}$ is calculated.

Step 6 The target detection probability $P_{d}$ of the echo signals with different SNRs is calculated. The process of 
obtaining the test statistics in this stage is the same as that of the threshold stage, but the observed signal model needs to add different echo signals to obtain the simulation data with a certain SNR. By comparing the test statistics with the threshold, the detection probability $P_{d}$ of the range distributed target under each SNR can be obtained and the location of the target is determined by (14).

From the discussion above we can know that the new search method of the detection window for the proposed detector is more flexible than that of the classical EI detector. Furthermore, it can be seen from Step 3 that comparing with the search mode of the traditional EI detector, the position and size of the detection window in the new search mode are more accurate, which provides more accurate position information for the target tracking, and greatly improves the detection performance. The flow chart of the proposed search method is shown in Fig. 1.

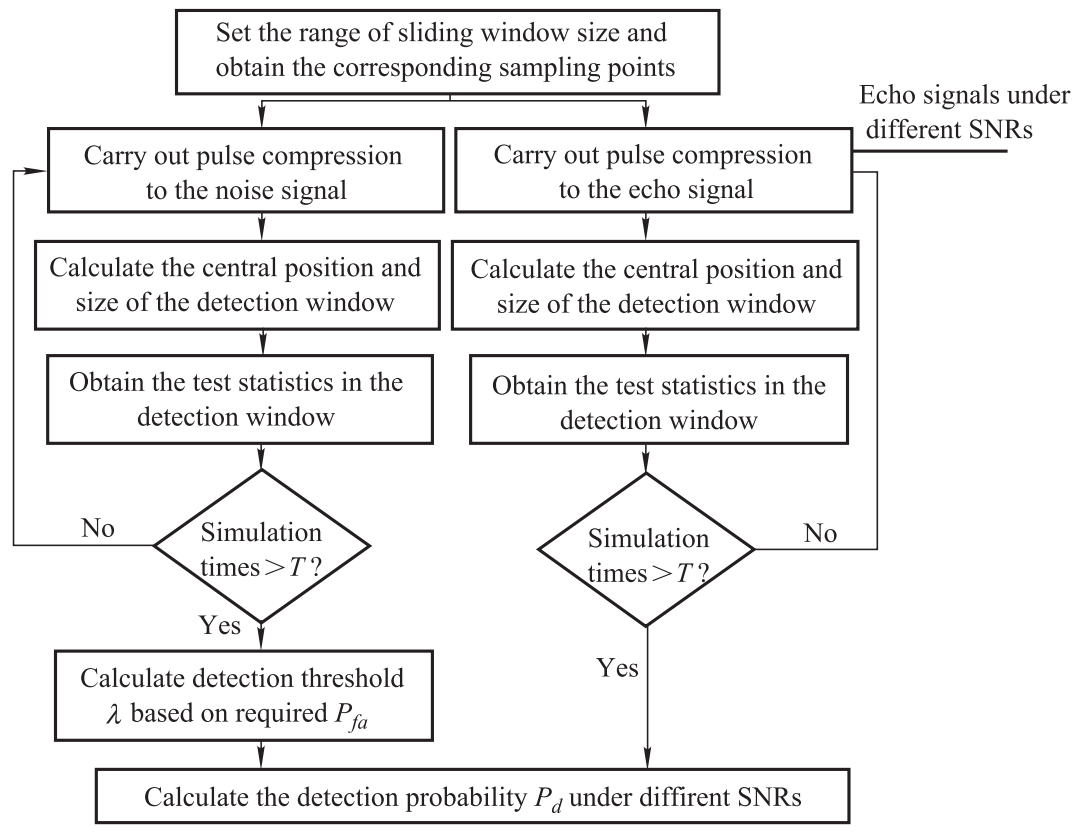

Fig. 1 Novel search method of detection window

\subsection{Optimized threshold setting method}

During the actual detection, the distribution of scattering points in different echoes will change with the transforms of target motion and radar angles of view. After searching the detection windows for each echo signal, their sizes obtained by different echo signals are often different, so that the test statistics $\Lambda$ will be quite different and unstable. The test statistic $\Lambda$ varies in a wide range with detection window sizes. However, when the detection background environment is invariant, the threshold $\lambda$ for the traditional detector is obtained by the unstable statistics, and it is unique and invariant. Therefore, when comparing the test statistics $\Lambda$ of each echo with the same threshold $\lambda$, the credibility and correctness of the results will not be optimistic.

In order to improve the detection performance, it is necessary to increase the stability of the test statistics $\Lambda$ as much as possible, and optimize the setting of the detection threshold $\lambda$. In this case, the problem that the actual length of the detection window is not consistent with the window length occupied by test statistics of different echoes will be solved. In this way, the threshold $\lambda$ is set more appropriately.

Firstly, the test statistics of the traditional EI detector are divided by the detection window size $N$ as the novel test statistics to represent the average power in the detection window, that is, the test statistics in the sense of average power can be expressed by

$$
\Lambda_{1}=\frac{2}{N \sigma^{2}} \sum_{n=1}^{N}\left|x_{n}\right|^{2}
$$

where $N$ is the number of sampling points in the detection window under the new search method, $\sigma^{2}$ is the noise average power, and $x_{n}$ is the echo signals of the $n$th range cell.

Compared to simple energy accumulation, this averaging process can constrain the fluctuation range of the test statistic $\Lambda_{1}$, which is beneficial to accomplish for hardware.

Note that the test statistic $\Lambda_{1}$ in the mean sense does not imply that a fixed-length sliding window can be used to search the detection window position. In fact, even if 
the average test statistic is used, the sizes of the detection window will still have an influence with the average test statistic. Thus, the average test statistic does not eliminate the influence of the different detection window lengths on the reliability of the threshold setting and detection performance.

Actually, the average test statistic of the smaller sliding window is more unstable than that of the longer sliding window. Because the sliding window length is too small, the average test statistic is not enough to smooth out the random outliers in the noise, resulting in degraded detection performance. That is, test statistics under this average concept is still related to the detection window length. Therefore, after using the test statistic of (16), the detection threshold is also needed to be further optimized in this paper.

The specific method is that a detection threshold for each possible sliding window is calculated, according to the possible size of the target, and then stores it in the current background environment. Therefore, when the target is detected, according to the length of the detection window, the threshold value can be determined and then used to perform a test decision. This method makes it possible that the ultimate threshold, which is always from the prestored thresholds, is calculated by the detection window with the same length. Thus, the inaccuracy of the judg- ment results caused by the sensitivity of test statistics to the window length can be eliminated, and the detection performance of the proposed detector can be improved.

The specific design steps for the optimized detection threshold are as follows:

Step 1 The current pulse data is simulated according to the observed signal model, and then pulse compression is performed. The noise-only data is included in the observed signal model to obtain the detection threshold.

Step 2 The sliding window is moved within the entire range gate according to the sliding steps, and the average test statistic for each sliding window is calculated.

Step 3 According to the Monte Carlo simulation times, Step 1 and Step 2 are repeated several times, and the test statistics of all sliding windows are obtained. Then the corresponding threshold $\lambda$ of the sliding window with the specific length is calculate based on the required false alarm probability $P_{f a}$.

Step 4 According to the step value of the sliding window length, all possible sliding windows are traversed, and Steps $1-3$ is repeated, thus all relevant detection threshold $\lambda$ are obtained.

The flow chart of the wideband target detection method for optimizing the detection threshold is graphically visualized in Fig. 2.

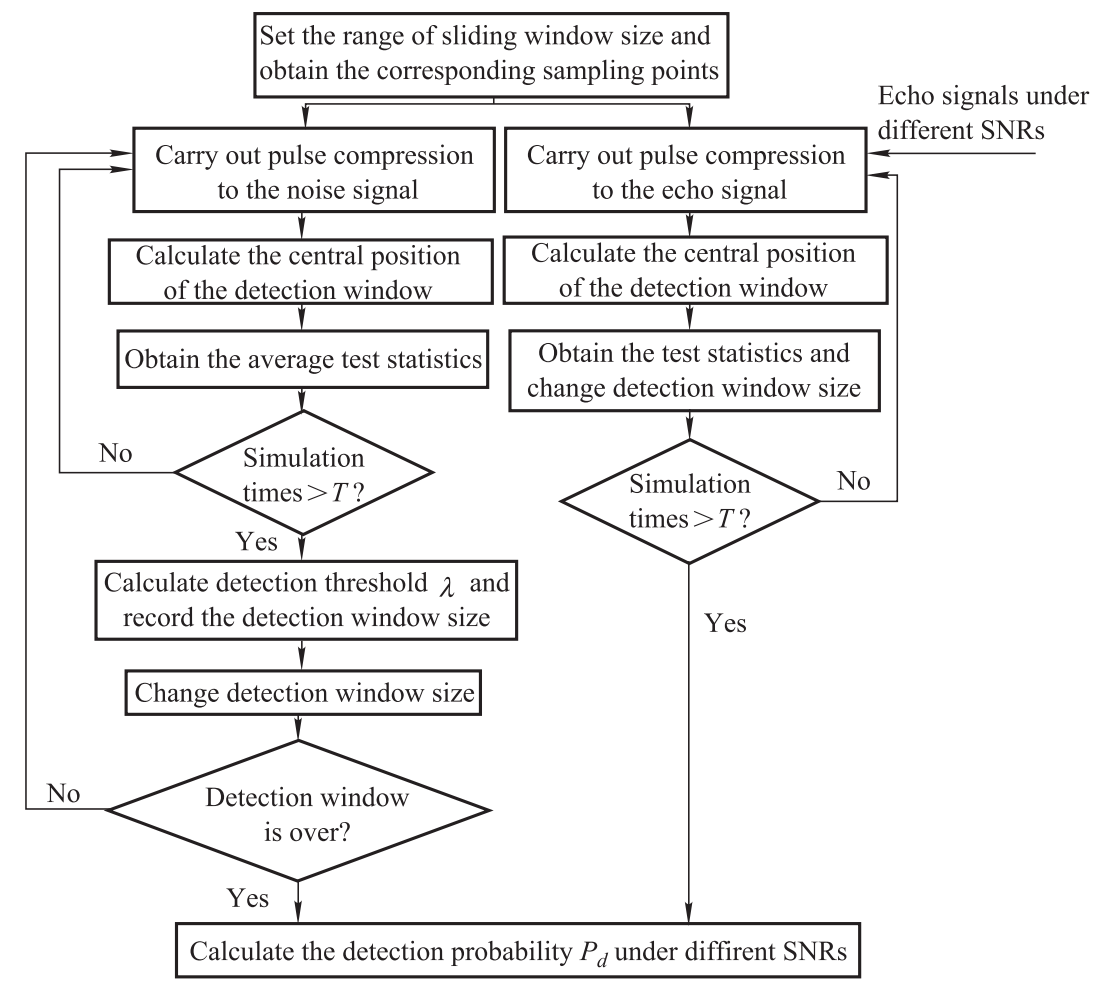

Fig. 2 Optimized method of threshold setting 
In this section, performance assessments of detectors are carried out. These five detectors are the traditional EI detector, the EI detector under the new detection window search method (Detector A), the EI detector for optimizing the detection threshold setting, including the detector with the traditional searching method of detection window (Detector B) and the detector with the novel search method (Detector C), and the scatterer density dependent GLRT (SDD-GLRT) detector (Detector D), respectively.

In the simulations, it is assumed that the radar transmits a train of linear frequency modulation (LFM) pulses

$$
s=A \cdot \operatorname{rect}\left(\frac{\tau}{T_{p}}\right) \mathrm{e}^{\mathrm{j} 2 \pi \cdot \frac{1}{2} k t^{2}}
$$

where frequency modulation (FM) slope is $k=\frac{B}{\tau}, B$ is the radar bandwidth, $\tau$ is the pulse duration, the noise is zero-mean complex Gaussian signals, then the model of the observed signal is

$$
x[n]=s[n]+w[n], \quad n=0,1, \ldots, N-1
$$

where $s[n]$ is the target echo and $w[n]$ is Gaussian signal, respectively, $N$ is the number of samples in the entire range gate. The related parameter settings in the simulation are shown in Table 1. Notice that distribution locations of scattering points are expressed by the normalized distances. The signal model and detector parameters of the other four kinds of detectors in this paper are the same as that of Detector A, and related parameters of Detector A are list in Table 2.

Table 1 Simulation parameters

\begin{tabular}{lcc}
\hline \multicolumn{2}{c}{ Parameter } & Value \\
\hline Noise power/dBW & Signal amplitude/V & 40 \\
\hline \multirow{5}{*}{ Radar parameters } & Bandwidth/MHz & 1 \\
& Pulse duration $/ \mu \mathrm{s}$ & 500 \\
& Carrier frequency/GHz & 10 \\
& Pulse repetition frequency/Hz & 10 \\
\hline \multirow{4}{*}{ Target parameters } & 200 \\
\hline & Initial range to radar/m & 5000 \\
& Radial velocity/(m/s) & 0 \\
& Target reality radial length/m & 30
\end{tabular}

10

$0.01,0.1,0.15,0.3$, $0.31,0.7,0.71,0.72$, $0.9,0.91$

Distribution location

\begin{tabular}{|c|c|c|}
\hline & Amplitude/V & $\begin{array}{l}20,30,30,30,20, \\
20,20,50,40,20\end{array}$ \\
\hline \multirow{3}{*}{$\begin{array}{l}\text { Tracking gate } \\
\text { parameters }\end{array}$} & Width/us & 30.2 \\
\hline & Starting time/ $\mu \mathrm{s}$ & 28.33 \\
\hline & Ending time/ $\mu \mathrm{s}$ & 58.53 \\
\hline
\end{tabular}
$20,30,30,30,20$,

In the following experiments, we assume that average false alarm time $T_{f a}$ is $50 \mathrm{~s}$, then

$$
P_{f a}=\frac{P R T}{T_{f a}}=\frac{1}{P R F \cdot T_{f a}}=1 \times 10^{-4} .
$$

Table 2 Simulation parameters of Detector $\mathbf{A}$

\begin{tabular}{cc}
\hline Parameter & Value \\
\hline Minimum length of searching window/m & 20 \\
Maximum length of searching window/m & 40 \\
Position stepper of sliding window & 4 \\
Length stepper of sliding window & 2 \\
\hline
\end{tabular}

Given the false-alarm probability $P_{f a}$, the NeymanPearson criterion is applied to obtain the detection threshold $\lambda$ based on the Monte Carlo method, where the number of Monte Carlo trials is $7 \times 10^{5}$. In addition, 300 independent experiments are conducted at each SNR level. The detection performance carves of five detectors are shown in Fig. 3 and Fig. 4.

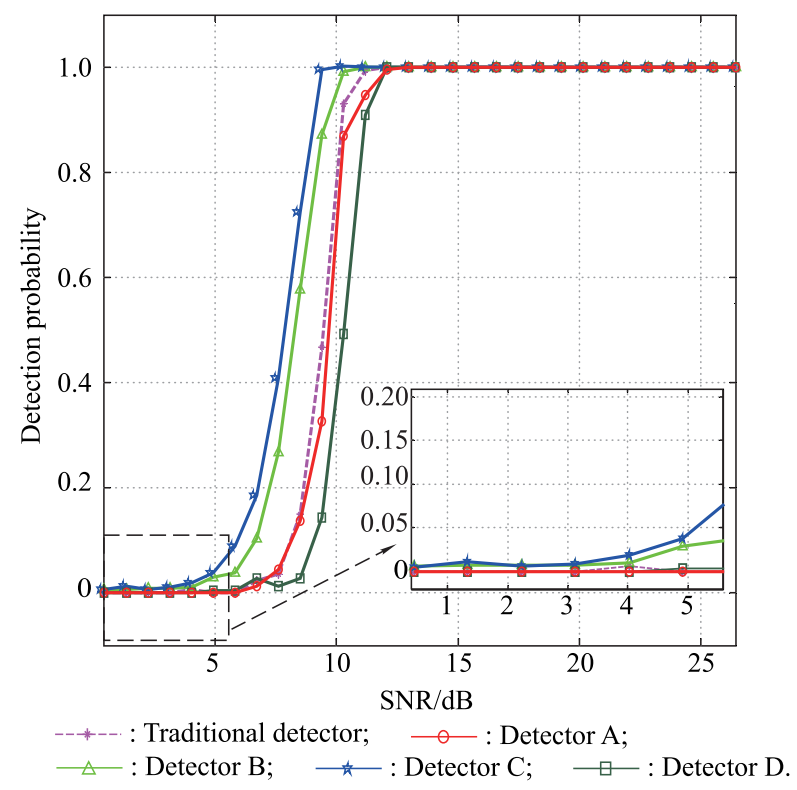

Fig. 3 Detection probability of the five detectors

As shown in Fig. 3, detection performance of Detector $\mathrm{C}$ is superior to the SDD-GLRT detector, Detector B and the traditional EI detector, which proves the effectiveness of the optimized detection threshold method, and also agrees with the theoretical analysis. In addition, the detection probability of Detector $\mathrm{C}$ is higher than that of Detector $\mathrm{B}$ by $1 \mathrm{~dB}$ collectively, which is approved that the novel searching manner of the detection window precedes the traditional searching manner.

Fig. 4 shows the position of the static target detected by the above five kinds of detectors. In Fig. 4(a), the traditional EI detector is the earliest one appearing missing detection, and the target position detected by Detector B is more accurate than the traditional EI detector, because Detector B has a better performance as shown in Fig. 3. In Fig. 4, four figures above show that the target position detected by Detector B and Detector C both change in a large scope. Because Monte Carlo simulation times 
become larger $\left(1 \times 10^{4}\right)$ for each SNR, the false alarm rate is $P_{f a}=1 \times 10^{-4}$, so the false alarm becomes more often. Fig. 4(c) and Fig. 4(d) show the comparison of the target position between Detector B and Detector C, both two detectors use the optimized threshold method, but Detector $\mathrm{B}$ uses the traditional searching method of the detection window while Detector $\mathrm{C}$ utilizes the optimized searching method of the detection window. If Fig. 4 and Fig. 3 are synthesized, it is obvious that the performance of Detector $\mathrm{C}$ is better.

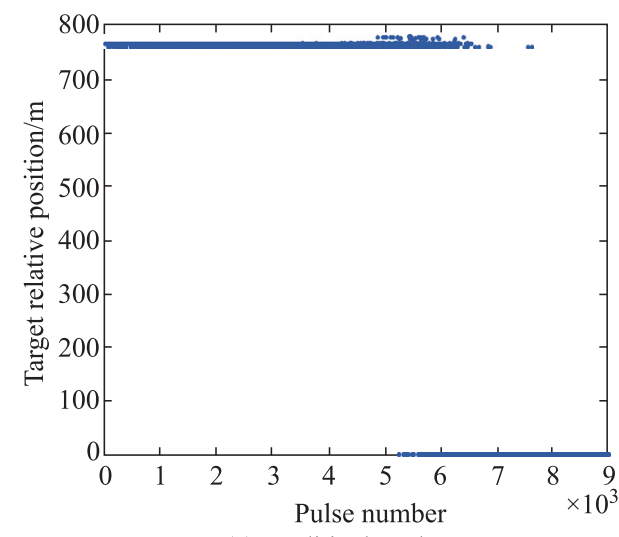

(a) Traditinal EI detector

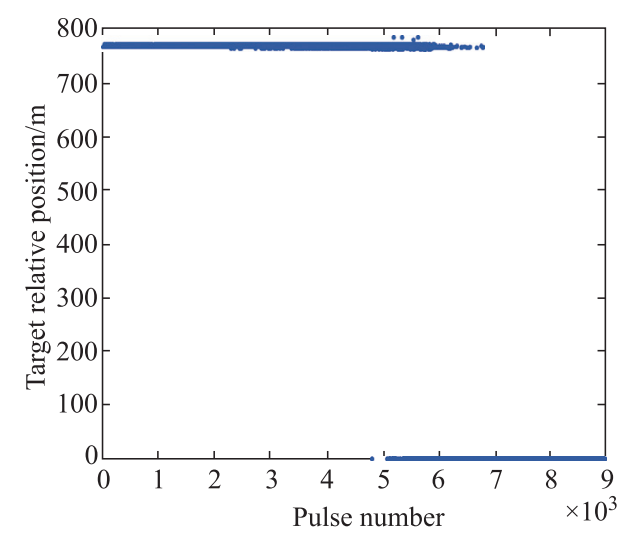

(b) Detector A

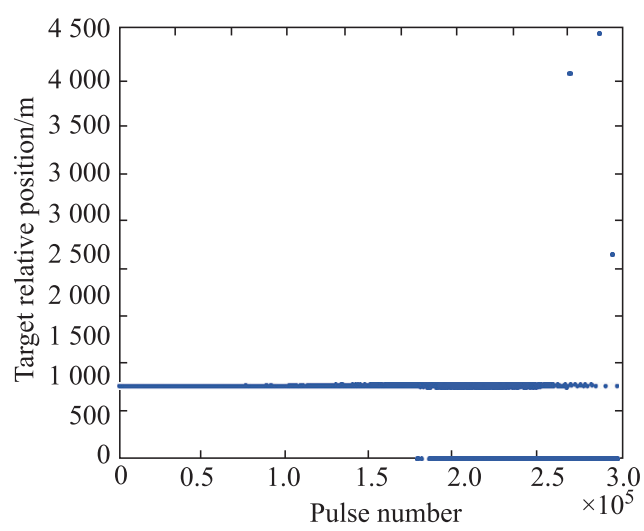

(c) Detector B

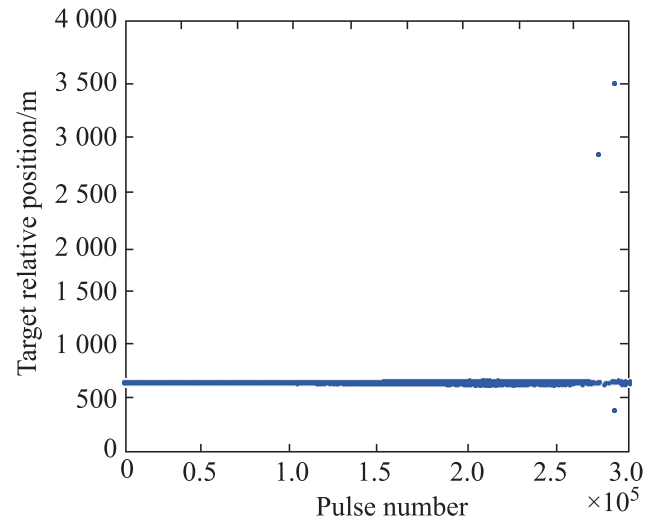

(d) Detector C

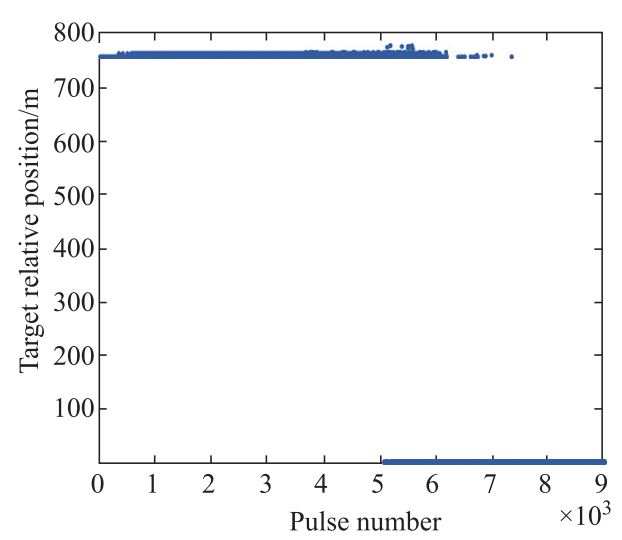

(e) SDD-GLRT detcctor

Fig. 4 Target position measured by detectors

In this paper, in order to verify the effectiveness of the proposed detector, another simulation with different parameters $N(N=60)$ and $K(K=6)$ is given, distribution location are $0.1,0.55,0.21,0.81,0.4,0.95$, and amplitudes are $15,45,20,55,75,20$, the other parameters are listed in Table 1 . The detection performance carves of the five detectors are shown in Fig. 5.

As shown in Fig. 5, detection performance of Detector C and Detector B outperforms other three detectors even in low SNR. With the same detection probability, Detector C and Detector B have the lowest requirement for SNR. More specifically, when the detection probability is 0.4 , SNR requirements of the traditional detector, Detector A, Detector B, Detector $\mathrm{C}$ and Detector D are $12 \mathrm{~dB}, 13 \mathrm{~dB}$, $7.47 \mathrm{~dB}, 7.49 \mathrm{~dB}$, and $11 \mathrm{~dB}$, respectively. In a word, the results above indicate that the proposed detector, combined with the novel searching method of the detection window and the optimized threshold setting method, greatly improves the detection probability compared with other detectors in this paper.

In conclusions, the proposed detector outperforms the conventional detectors. Because the optimized searching method of the detection window could get a more suit- 
able detection window size and get a more accurate target position. Furthermore, the optimized threshold setting method could get more suitable threshold. Thus, by combining these two methods, the detection performance can be improved remarkably.

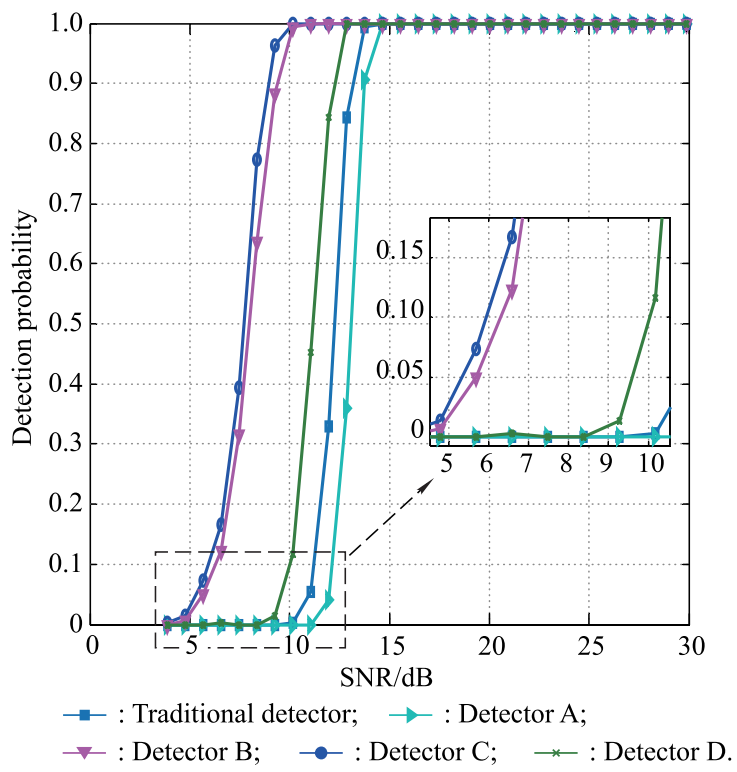

Fig. 5 Detection probability of the five detectors

\section{Conclusions}

The EI detector has low complexity and can be implemented easily in practical scenarios. Based on the EI detector, a kind of high-performance EI detector for the rangedistributed target in the Gaussian environment is proposed in this paper. Firstly, the considered model of distributed targets is derived, under both the $H_{0}$ and $H_{1}$ hypotheses. Secondly, the novel searching method of the detection window and the optimized method of the threshold setting are provided. The theory indicates that the proposed detector does not need the prior knowledge about the spatial distribution of the target scattering centers. Finally, the effectiveness of the proposed detector has been verified by Monte Carlo simulations. Numerical results demonstrate that the proposed detector is more suited for the detection of distributed targets than the traditional EI detector and the SDD-GLRT detector even in low-SNR scenarios.

\section{References}

[1] ROBEY F C, FUHNNANN D R, KELLY E J, et al. A CFAR adaptive matched filter detector. IEEE Trans. on Aerospace and Electronic Systems, 1992, 28(1): 208-216.

[2] KELLY E J. An adaptive detection algorithm. IEEE Trans. on Aerospace and Electronic Systems, 1986, 22(2): 115-127.

[3] FARINA A, SCANNAPIECO F, VINELLI F. Target detection and classification with very high range resolution radar. Proc. of the International Conference on Radar, 1989: 20-25.
[4] FARINA A, SCANNAPIECO F, VINELLI F. Target detection and classification with polarimetric high resolution range radar. BOERNER W M, ed. Direct and inverse methods in radar polarimetry, Part I. Netherlands: Kluwer Academic Publishers, 1989: $1021-1041$.

[5] MOON T T, BAWDEN P J. High resolution RCS measurements of boats. IEE Proceedings F - Radar Signal Processing, 1991, 138(3): $218-222$.

[6] YANG X L, WEN G J, MA C H, et al. CFAR detection of moving range-spread target in white Gaussian noise using waveform contrast. IEEE Geoscience and Remote Sensing Letters, 2016, 13(2): 282-286.

[7] BERIZZI F, MARTORELLA A, CAPRIA A, et al. A contrast based algorithm for synthetic range-profile motion compensation. IEEE Trans. on Geoscience and Remote Sensing, 2008, 46(10): $3053-3062$.

[8] ZHANG S X, XING M D, XIA X G, et al. Robust clutter suppression and moving target imaging approach for multichannel in azimuth high-resolution and wide-swath synthetic aperture radar. IEEE Trans. on Geoscience and Remote Sensing, 2015, 53(2): $687-709$.

[9] MARTORELLA M, BERIZZI F, HAYWOOD B. A contrast maximization based technique for 2D ISAR autofocusing. IEE Proceedings - Radar, Sonar and Navigation, 2005, 152(4): $253-262$.

[10] JIAN T, HE Y, SU F, et al. Robust detector for range-spread targets in non-Gaussian background. Journal of Systems Engineering and Electronics, 2012, 23(3): 355 - 363.

[11] SEKINE H. Weibull, log-Weibull and K-distributed ground clutter modeling analyzed by AIC. IEEE Trans. on Aerospace and Electronic Systems, 2001, 37(3): 1108 - 1113.

[12] DE MAIO A, ORLANDO D. Adaptive radar detection of a subspace signal embedded in subspace structured plus Gaussian interference via invariance. IEEE Trans. on Signal Processing, 2016, 64(8): 2156-2167.

[13] CIUONZO D, MAIO A D, ORLANDO D. On the statistical invariance for adaptive radar detection in partially homogeneous disturbance plus structured interference. IEEE Trans. on Signal Processing, 2017, 65(5): 1222-1234.

[14] CONTE E, DE MAIO A, RICCI G. GLRT-based adaptive detection algorithms for range-spread targets. IEEE Trans. on Signal Processing, 2001, 49(7): 1336-1348.

[15] XIAO L, LIU Y, HUANG T, et al. Distributed target detection with partial observation. IEEE Trans. on Signal Processing, 2018, 66(6): $1551-1565$.

[16] WU D, ZHU D, SHEN M W, et al. Statistical analysis of monopulse-synthetic aperture radar for constant false-alarm rate detection of ground moving targets. IET Radar, Sonar and Navigation, 2015, 9(6): 641-652.

[17] LIU W, LIU J, HUANG L, et al. Distributed target detectors with capabilities of mismatched subspace signal rejection. IEEE Trans. on Aerospace and Electronic Systems, 2017, 53(2): $888-900$.

[18] LIU W, LIU J, DU Q, et al. Distributed target detection in partially homogeneous environment when signal mismatch occurs. IEEE Trans. on Signal Processing, 2018, 66(14): 3918 3928.

[19] GAO Y, LI H, HIMED B. Knowledge-aided range-spread target detection for distributed MIMO radar in nonhomogeneous environments. IEEE Trans. on Signal Processing, 2017, 65(3): $617-627$.

[20] ZHANG X W, LI M, ZUO L, et al. Adaptive subspace detection for wideband radar using sparsity in sinc basis. IEEE Trans. on Geoscience and Remote Sensing, 2014, 11(11): 1916- 1920. 
[21] MARTORELLA M, BERIZZI F, HAYWOOD B. A contrast maximization based technique for 2D ISAR autofocusing. IEE Proceedings - Radar, Sonar and Navigation, 2005, 152(4): $253-262$.

[22] MAIO A D, CONTE E. Adaptive detection in Gaussian interference with unknown covariance after reduction by invariance. IEEE Trans. on Signal Processing, 2010, 58(6): $2925-$ 2934.

[23] LI N, CUI G, KONG L, et al. Moving target detection for polarimetric multiple-input multiple-output radar in Gaussian clutter. IET Radar, Sonar and Navigation, 2015, 9(3): $285-$ 298.

[24] CHEN P, ZHENG L, WANG X D. Moving target detection using colocated MIMO radar on multiple distributed moving platforms. IEEE Trans. on Signal Processing, 2017, 65(17): $4670-4683$.

[25] CUI G L, YU X X, KONG L J. Exact distribution for the product of two correlated Gaussian random variables. IEEE Signal Processing Letters, 2016, 23(11): $1662-1666$.

[26] CAROTENUTO V, MAIO A D, ORLANDO D, et al. Adaptive radar detection using two sets of training data. IEEE Trans. on Signal Processing, 2018, 66(7): $1791-1801$.

[27] AUBRY A, MAIO A D, PALLOTTA L, et al. Radar detection of distributed targets in homogeneous interference whose inverse covariance structure is defined via unitary invariant functions. IEEE Trans. on Signal Processing, 2013, 61(20): 49494961.

[28] BUDILLON A, SCHIRINZI G. Performance evaluation of a GLRT moving target detector for TerraSAR-X along-track interferometric data. IEEE Trans. on Geoscience and Remote Sensing, 2015, 53(6): 3350-3360.

[29] HAO C, YANG J, MA X, et al. Adaptive detection of distributed targets with orthogonal rejection. IET Radar, Sonar and Navigation, 2012, 6(6): 483-493.

[30] CONTE E, MAIO D A, RICCI A, et al. CFAR detection of distributed targets in non-Gaussian distribution. IEEE Trans. on Aerospace and Electronic Systems, 2002, 38 (2): 612-621.

[31] CIUONZO D, ORLANDO D, PALLOTTA L. On the maximal invariant statistic for adaptive radar detection in partially homogeneous disturbance with persymmetric covariance. IEEE Trans. on Signal Processing, 2016, 23(12): 1830-1834.

[32] CIUONZO D, MAIO A D, ORLANDO D. On the statistical invariance for adaptive radar detection in partially homogeneous disturbance plus structured interference. IEEE Trans. on Signal Processing, 2017, 65(5): $1222-1234$.

[33] DONG Y, LIU M, LI K. Adaptive direction detection in deterministic interference and partially homogeneous noise. IEEE Signal Processing letters, 2017, 24(5): 599-603.

[34] ABRAMOVICH Y I, BESSON O. Fluctuating target detection in fluctuating K-distributed clutter. IEEE Signal Processing Letters, 2015, 22(10): $1791-1795$.
[35] YANG X L, WEN G J, MA C H, et al. CFAR detection of moving range-spread target in white Gaussian noise using waveform contrast. IEEE Geoscience and Remote Sensing Letters, 2016, 13(2): $282-286$.

[36] SANGSTON K J, GINI F, GRECO M S. Coherent radar target detection in heavy-tailed compound-Gaussian clutter. IEEE Trans. on Aerospace and Electronic Systems, 2012, 48(1): $64-$ 77.

\section{Biographies}

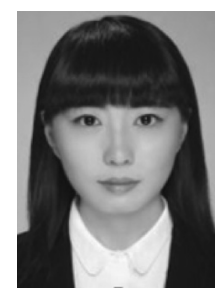

CHANG Jiayun was born in 1989. She received her master degree from Beijing Institute of Technology (BIT), China, in 2016. Currently, she is pursuing her Ph.D. degree in the School of Information and Electronics, BIT. Her research interests include automatic target recognition and radar signal processing.

E-mail: 824400828@qq.com

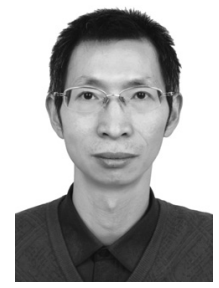

FU Xiongjun was born in 1978. He received his B.E. degree and Ph.D. degree from Beijing Institute of Technology (BIT), China, in 2000 and 2005 respectively. He is currently the vice dean of the School of Information and Electronics, BIT, and an associate professor and $\mathrm{PhD}$ supervisor with BIT. His current research interests include radar system, radar signal processing, waveform design, and automatic target recognition.

E-mail: fuxiongjun@bit.edu.cn

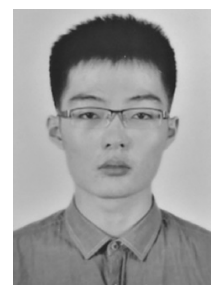

JIANG Wen was born in 1991. He received his master degree from Zhengzhou University, China, in 2016. Currently, he is pursuing his Ph.D. degree in the School of Information and Electronics, Beijing Institute of Technology. His research interests include radar signal processing and radar signal sorting.

E-mail: jwen912@126.com

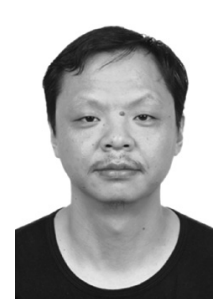

XIE Min was born in 1976. He received his Ph.D. degree from Beijing Institute of Technology (BIT), China, in 2006. Currently, he is a lecturer of the School of Information and Electronics, BIT. His research focuses on high-speed real-time signal processing, radar signal processing, signal acquisition and storage.

E-mail: xiemin@bit.edu.cn 